\title{
Contribution à l'amélioration de la qualité de la consultation post natale : une étude d'intervention au Centre de Santé Communautaire et Universitaire de Konobougou, Mali
}

\section{Contribution to improving the quality of postnatal care: an intervention study at the Community and University health Center of Konobougou, Mali}

\author{
Coulibaly $\mathrm{MB}^{1^{*}}$, Traoré $\mathrm{S}^{2}$, Coulibaly $\mathrm{KB}^{3}$, Konaté $\mathrm{A}^{4}$, Bengaly $\mathrm{I}^{5}$, Maïga $\mathrm{M}^{6}$, Touré $\mathrm{M}^{7}$
}

\section{DOI : 10.53318/msp.v11i1.1888}

1. Centre de santé communautaire et universitaire de Konobougou (Ségou-Mali)

2. Centre de Santé de Référence de Barouéli (Ségou-Mali)

3. Centre de santé communautaire et universitaire de Sanoubougou 2 (Sikasso-Mali)

4. Centre de santé communautaire et universitaire de Segué (Koulikoro-Mali)

5. Centre de santé communautaire et universitaire de Koniakary (Kayes-Mali)

6. Communauté Locale d'Enseignement pour les Femmes et les Filles en Santé (Bamako-Mali)

7. Service de gynéco-obstétrique CHU hôpital du Mali (Bamako-Mali)

*Auteur correspondant : Dr Mamadou Bayo Coulibaly, spécialiste en médecine de famille/médecine communautaire, Directeur technique du CSCom U de Konobougou, Chargé d'encadrement clinique D.E.S médecine de famille/médecine communautaire (Mali). bayo_coul@yahoo.fr ; Cel : 0022376067882

\section{Résumé}

Introduction : Les six semaines du postpartum constituent une période à risque de morbidité et de mortalité maternelle et néonatale. Pour prévenir ces risques il est recommandé des visites postnatales de la mère et le nouveau-né. Le but de ce travail était de contribuer à l'amélioration de la qualité de la consultation post natale dans le centre de santé communautaire et universitaire (CSCom-U) de Konobougou. Matériel et méthodes : II s'agissait d'une étude interventionnelle transversale répétée allant du 01 juillet au 30 novembre 2020 au CSCom-U de Konobougou. Résultats : Nous avons introduit dans cette étude 244 femmes en post partum dont la moitié à l'évaluation initiale et l'autre moitié à l'évaluation finale plus sept agents de santé. La connaissance des signes de danger pour les femmes elles-mêmes et pour les nouveau-nés a respectivement évoluée de $67,2 \%$ à $91,8 \%$ des cas et de $65,6 \%$ à $95,1 \%$ des cas. Seulement $14,3 \%$ des nouveau-nés étaient examiné à l'évaluation initiale contre $100 \%$ à l'évaluation finale grâce au plan d'action. Le résultat de l'examen physique n'était pas annoncé aux femmes dans $71,4 \%$ des cas à l'évaluation initiale contre $0 \%$ l'évaluation secondaire. Conclusion : Le plan d'action a permis de corriger les insuffisances observées au cours de l'évaluation initiale, d'améliorer la compétence des agents de santé et le niveau de connaissance des femmes.

Mots clés : Amélioration qualité, consultation post natale, Konobougou, Mali

Abstract
Introduction: The period of the first six weeks postnatal is a period of risk if no consultation for mothers and their newborns has been provided. The aim of this work was to improve the quality of post-natal consultation in the community and university health center of Konobougou. Material and methods: This was a cross-sectional interventional study repeated at the health center from July 1 to November 30, 2020. Results: We introduced into this study 244 women in postpartum, who's half at the initial assessment and the other half at the final assessment plus seven health workers. Knowledge of danger signs for women themselves and for newborns increased respectively from $67.2 \%$ to $91.8 \%$ of cases and from $65.6 \%$ to $95.1 \%$ of cases. Only $14.3 \%$ of newborns were examined at the initial assessment against $100 \%$ at the final assessment using the action plan. The results of the physical examination were not announced to women in $71.4 \%$ of cases at the initial assessment compared against $0 \%$ at the secondary assessment. Conclusion: The action plan made it possible to correct the shortcomings observed during the initial assessment, improve the skills of health workers and the level of knowledge of women.

Keywords: Quality improvement, post-natal consultation, Konobougou, Mali

\section{Introduction}

La santé maternelle et infantile est au cœur des objectifs du millénaire pour le développement (OMD 4 et 5) (1). L'éducation pour la santé en périnatalité peut être définie comme un processus centré sur les femmes visant à augmenter leur autonomie et à faciliter l'acquisition ou le maintien des compétences dont elles ont besoin pour gérer et s'adapter aux changements inhérents à la maternité. Ce processus concerne donc la grossesse, la période de la naissance, le post-partum et l'enfant (2). Chaque année dans le monde, environ 350.000 femmes meurent en couches et environ 8 millions d'enfants meurent avant leur cinquième anniversaire dont plus de $99 \%$ surviennent dans les pays en développement (1). Les décès maternels ne sont pas uniformément répartis dans le monde, et le risque obstétrical est de loin le plus élevé en Afrique subsaharienne (3). Deux tiers de ces décès maternels ont lieu après la naissance, pendant la période du post partum $(3,4)$.

Le post-partum couvre la période allant de 2 heures à 6 semaines suivant l'accouchement (4). Pour les mères, c'est une période riche en bouleversements tant physiologiques, qu'anatomiques et hormonaux $(4,5)$. La période des six premières semaines postnatales est une période à risque si aucune consultation pour les mères et 
leurs nouveau-nés n'a été fournie (6). Le post-partum constitue pour les cliniciens une occasion unique et privilégiée d'aborder la santé physique, psychique et sociale des femmes et de leur enfant (7). La consultation post natale (CPON) permet de clôturer le suivi de grossesse, jouant un rôle de synthèse et d'information de la patiente (7). Elle reste l'un des moyens les plus importants pour réduire la mortalité maternelle et néonatale (1). L'entretien post-natal précoce est un nouvel outil de prévention primaire ayant pour objectif d'améliorer le bien-être global des femmes, des nouveau-nés et des familles, basé sur l'écoute, le soutien et l'empathie, s'inscrivant dans une pratique éducative en réseau (8). Les soins postnatals sont centrés sur les besoins spécifiques de la femme et du nouveau-né (4).

Selon la 6ième édition de l'enquête démographique et de santé du Mali (EDSM VI) 2018, le taux de mortalité maternelle au Mali est de 325 pour 100000 naissances vivantes ; le taux de de mortalité néonatale est de 101 pour 1000 naissances vivantes (9). Cet état de fait a été attribué essentiellement à : l'insuffisance services de santé de qualité et de ressources humaines pour couvrir les besoins (10). Dans le souci de fournir des prestations de qualité correspondant aux besoins prioritaires des populations, une supervision interne effectuée par le personnel interne du centre au centre de santé communautaire et universitaire a constaté des insuffisances dans la réalisation de la consultation postnatale au centre de santé communautaire et universitaire de Konobougou. Nous avons ainsi mené ce travail pour contribuer à l'amélioration de la qualité de la consultation post natale (CPON) dans cette structure de santé.

\section{Matériel et méthodes}

II s'agissait d'une étude interventionnelle transversale répétée au centre de santé communautaire et universitaire de Konobougou. Elle s'est déroulée en cinq mois allant du 01 juillet au 30 novembre 2020 .

Cette étude s'est réalisée en trois phases. La première phase a été une analyse de situation et l'élaboration d'un plan d'action de la CPON ; la seconde a été consacrée à la mise en œuvre du plan d'action par la personne impliquée dans la réalisation de la CPON et la troisième a été l'évaluation finale. La taille de l'échantillon était de 244 femmes en post partum et 7 agents de santé. Nous avons pris toutes les femmes vues en CPON pendant les deux premiers mois à l'évaluation initiale soit 122 . Ce qui nous a amené à prendre le même nombre à l'évaluation finale qui a duré deux mois aussi.

Ont été inclut dans notre étude, les accouchées récentes de moins de 42 jours ayant acceptée de participer à l'étude. N'ont pas été inclut dans l'étude les femmes dont l'état de santé ne permettait pas l'inclusion.

Au cours de notre étude la qualité de consultation post natale était fonction du respect des procédures politiques normes de consultation (selon les PNP : Procédures Politiques et Normes),
Les paramètres étudiés étaient :

- La disponibilité d'une salle de CPON, du personnel soignant, des supports d'enregistrement, des matériels et des consommables ;

- Chez le personnel : la qualification du personnel, l'explication du déroulement de la CPON, la prise des paramètres de la femme et des nouveau-nés, le respect de l'étape de l'examen physique, la vérification (du statut vaccinal de l'enfant, de la montée laiteuse et de l'état des lochies), l'examen du nouveau-né ;

- Chez les femmes en post partum : les caractéristiques sociodémographiques des femmes en post partum, la connaissance des femmes (sur les signes de danger pour elles-mêmes et le nouveau-né, la fréquence de la tétée, l'allaitement exclusif, la position de la mère et du nouveau-né pendant l'allaitement, la succion efficace) et la satisfaction des femmes pour le service de CPON.

Les données ont été recueillies sur une fiche d'enquête plus une grille puis saisies et analysées par le logiciel SPSS 22. Cette étude a été réalisée dans le respect des règles d'éthiques.

\section{Résultats}

L'étude a concerné 244 femmes en post partum dont 122 à l'évaluation initiale et 122 à la fin de l'étude et sept agents de santé.

La tranche d'âge 20 à 29 ans a été la plus représentée avec $47,5 \%$ des cas et l'âge moyen était de 25,12 ans avec des extrêmes 17 et 45 ans à l'évaluation initiale contre $52,5 \%$ pour les 20 à 29 ans pendant la seconde évaluation avec une moyenne d'âge de 28,43 et des extrêmes 16 et 43 ans (Tableau I).

Nous avons noté l'existence d'une salle de CPON propre, éclairée et bien aérée. Les supports primaires, les matériels, les consommables et le personnel soignant étaient disponibles à 100\% pendant les périodes d'étude. Le personnel non qualifié représentait $57,1 \%$ des cas versus $42,9 \%$ du personnel qualifié pendant les deux études (figure 1). A l'évaluation initiale, 14,29\% seulement du personnel avaient reçu de formation sur la CPON à l'évaluation initiale contre $100 \%$ à la fin de l'étude.

A la première évaluation, $71,4 \%$ du personnel avaient expliqué le déroulement de la CPON contre à $85,7 \%$ des cas à l'évaluation finale. Les paramètres étaient pris chez toutes les femmes au cours des deux études. Les étapes de l'examen ont été respectées dans $85,7 \%$ des cas à la première évaluation contre $100 \%$ des cas à la seconde évaluation.

Le résultat de l'examen physique n'était pas annoncé aux femmes dans $71,4 \%$ des cas à l'évaluation initiale alors que pendant la seconde évaluation $85,7 \%$ de personnel a donné le résultat de l'examen à la femme soit un gain de $57,1 \%$.

La montée laiteuse a été vérifiée dans tous les cas pendant les deux évaluations soit $100 \%$ des cas. L'examen des organes génitaux a été réalisé dans $85,7 \%$ 
des cas à la première évaluation contre $100 \%$ des cas à l'évaluation secondaire. L'observation de lochies a progressé de $85,7 \%$ à $100 \%$ des cas entre les deux évaluations. La vérification de la prise de fer acide folique en post partum était la même pour les deux évaluations avec $71,4 \%$ des cas. Le conseil sur la planification familiale a été donné aux femmes en post partum dans $71,4 \%$ dans l'évaluation primaire contre $85,7 \%$ à l'évaluation finale.

Seulement $14,3 \%$ des nouveau-nés étaient examinés à l'évaluation initiale contre 100\% à l'évaluation finale (figure 2). Le statut vaccinal des enfants a été vérifié dans tous les cas au cours des deux évaluations par les soignants. Les femmes qui venaient dans un rayon de $5 \mathrm{~km}$ représentaient respectivement $95,1 \%$ et $91,8 \%$ des cas dans la première et seconde évaluation (Tableau I).

Les femmes non scolarisées représentaient respectivement $52,5 \%$ et $57,4 \%$ des cas au cours de la première et seconde évaluation (Tableau I). Les paucipares et les multipares représentaient respectivement $36,1 \%$ et $27,9 \%$ des cas dans l'évaluation initiale alors que dans la seconde évaluation $37,7 \%$ était des multipares et $36,1 \%$ des paucipares (Tableau I).

La bonne connaissance de la fréquence des tétées par les femmes était $98,4 \%$ des cas au cours des deux évaluations. La connaissance des signes de danger pour les femmes elles-mêmes et pour les nouveau-nés avait respectivement évolué de $67,2 \%$ à $91,8 \%$ des cas et de $65,6 \%$ à $95,1 \%$ des cas (Tableau II). La connaissance de la période de l'allaitement exclusif a progressé de $86,9 \%$ à $95,1 \%$ des cas.

La bonne position des mères et celle des nouveau-nés au cours de l'allaitement étaient connues respectivement dans $80,3 \%$ et $78,7 \%$ des cas à l'évaluation initiale contre $91,8 \%$ pour les deux cas à l'évaluation finale. La connaissance sur l'efficacité de la succion du nouveau-né par les mères était $80,3 \%$ contre $82 \%$ des cas respectivement dans l'évaluation initiale et secondaire.

Nous avons observé un gain des femmes qui étaient très satisfaites pour le service de la CPON de $62,7 \%$ à $80,3 \%$ des cas (Tableau III).

\section{Discussion}

Nous avons noté l'existence d'une salle de CPON propre, éclairée et bien aérée. Les supports primaires (registres de CPON), les matériels, les consommables et le personnel soignant étaient tous disponibles. Cela permettrait d'assurer la prise en charge correcte des femmes au cours de la CPON d'une part et serait une source de motivation d'autre part.

Plus de la moitié du personnel était non qualifié (matrone) (10). Cela démontrait qu'en milieu rural, les agents de santé qui n'ont pas de qualification jouaient un rôle non négligeable dans la pratique de consultation post natale. La 2ème consultation post natale a été assurée principalement par les gynécologues obstétriciens dans $14,9 \%$ et $85,1 \%$ des cas par les sages-femmes dans l'étude de Koné CS réalisée à Bamako en 2020 en milieu urbain (11). Alors qu'elle est réalisée par un médecin gynécologue obstétricien, gynécologue médical, généraliste ou une sage-femme en cas de grossesse normale et d'accouchement eutocique selon Sénat MV et al. et Doret $M(7,12)$. Les différences des données de notre étude et de celles de Koné CS, de Doret MV et de Sénat et al s'expliqueraient par notre lieu d'intervention en zone rurale ou il y avait moins de personnel qualifié.

La tranche d'âge 20 à 29 ans a été la plus représentée avec $47,5 \%$ des cas et l'âge moyen était de 25,12 ans avec des extrêmes 17 et 45 ans à l'évaluation initiale contre $52,5 \%$ pour les 20 à 29 ans pendant la seconde évaluation avec une moyenne d'âge de 28,43 et des extrêmes 16 à 43 ans. Une tendance similaire à l'évaluation initiale a été observée chez Elkhoudri $\mathrm{N}$ et al. ou l'âge variait de 15 à 49 ans avec une moyenne d'âge de 28,2 (1). Tandis qu'un âge moyen était de 31,4 ans dans le travail de Maamri $A$ et al., ce résultat était supérieur à ceux des deux évaluations de notre étude (13).

L'explication faite par les agents de santé sur le déroulement de la CPON a évolué de $71,4 \%$ à $85,7 \%$ des cas entre les deux évaluations. Le fait de donner des explications sur le déroulement de la CPON faciliterait la prise en charge des femmes tout en créant un sentiment de confiance entre le personnel et les femmes.

Les paramètres étaient pris chez toutes les femmes au cours des deux études. Les étapes de l'examen ont été respectées dans $85,7 \%$ des cas à la première étude contre $100 \%$ des cas à la seconde évaluation. La prise des paramètres et le respect des étapes de l'examen physique étaient capitales, ils permettraient de récolter tous les indices et de ne pas laisser passer certains éléments importants comme la tension artérielle, le poids, la température....). Alors que dans l'étude de Elkhoudri $\mathrm{N}$ et al., la prise de température le pouls ont été réalisées respectivement dans $40 \%$ et $11 \%$ des cas, celle de la pression artérielle dans $73 \%$ des cas (1). Tandis que tous les paramètres ont été évalués chez les patientes vues à la deuxième CPON dans le travail de Koné CS similaire à notre étude (11).

Le résultat de l'examen physique n'était pas annoncé aux femmes dans $71,4 \%$ des cas à l'évaluation initiale alors que pendant l'évaluation secondaire $85,7 \%$ de personnel a donné le résultat de l'examen à la femme soit un gain de $57,1 \%$. L'annonce du résultat de l'examen rassurerait la femme et l'amènerait à respecter davantage les mesures de prévention et de promotion en post partum.

Une sécrétion lactée a été observée dans tous les cas pendant les deux évaluations soit $100 \%$ des cas. Cela encouragerait la poursuite de l'allaitement maternel exclusif et la création de liens affectif mère-enfant.

L'examen des organes génitaux a été réalisé dans $85,7 \%$ des cas à la première évaluation contre $100 \%$ des cas à l'évaluation secondaire. L'observation de l'aspect des lochies a progressé de $85,7 \%$ à $100 \%$ des cas entre les deux évaluations. Cela permettrait de vérifier la présence ou non d'une lésion génitale ou d'une endométrite qui 
conduirait à leur prise en charge. Par contre notre résultat était supérieur à celui de Elkhoudri $\mathrm{N}$ et al. ou l'examen gynécologique et l'aspect des lochies n'ont été réalisé respectivement que dans $5 \%$ et $40 \%$ des cas (1).

Environ $71,4 \%$ des patientes avaient reçu des informations sur les méthodes contraceptives à l'évaluation initiale versus $85,7 \%$ à l'évaluation finale. Alors que Blangis $F$ et al. avaient trouvé $73,5 \%$ de patientes ayant reçu une contraception hormonale (14). Cependant, Sénat MV et al et la revue des praticiens avaient annoncé que les risques des grossesses rapprochées devraient être expliqués et la contraception discutée en fonction du projet familial $(7,15)$.

Dans notre étude seulement $14,3 \%$ des nouveau-nés ont bénéficié d'un examen médical à l'évaluation initiale contre $100 \%$ à l'évaluation finale. Leur statut vaccinal (BCG et polio 0) a été vérifié dans les deux évaluations à $100 \%$ des cas. L'examen médical et la vérification du statut vaccinal permettraient de s'assurer de bonne santé de celui-ci et de lutter contre les maladies infectieuses. Selon MV Sénat et al., la vaccination en post-partum immédiat pouvait prévenir la contamination de l'enfant en période postnatale (7).

La vérification de la prise orale de fer acide folique était retrouvée dans $71,4 \%$ des cas lors des deux évaluations en période post natale. Cela nous assurerait à éviter l'anémie du post partum par la prise du fer par les mères. Alors qu'une supplémentation martiale orale n'était réalisée que dans les cas d'anémie biologiquement prouvée selon Sénat MV et al. (7).

Les paucipares ont été la plus touchée $36,1 \%$ des cas dans l'évaluation initiale contre $37,7 \%$ des mulipares dans la seconde évaluation. Tandis que les paucipares étaient également les plus représentées $44,6 \%$ des cas dans le travail de Koné $\mathrm{C} S$, la même tendance semblable à notre résultat (11).

Les femmes non scolarisées représentaient respectivement $52,5 \%$ et $57,4 \%$ des cas au cours de la première et seconde évaluation. Alors que le taux d'analphabétisme chez les femmes était de $25,1 \%$ dans l'étude d'Elkhoudri $\mathrm{N}$ et al., ce résultat était différent au nôtre (1). Tandis que plus d'une patiente sur trois (33\%) n'était pas scolarisée dans le travail de Koné C S (11). La bonne connaissance de la fréquence des tétées par les femmes était $98,4 \%$ des cas au cours des deux évaluations. Cependant, pour Sénat MV et al, il n'était pas recommandé de respecter un espacement strict des tétées toutes les $4 \mathrm{~h}$ car cette attitude était associée à plus de difficultés de l'allaitement et des arrêts précoces (7). La connaissance des signes de danger pour femmes elles-mêmes et pour les nouveau-nés avait respectivement évoluée de $67,2 \%$ à $91,8 \%$ des cas et de $65,6 \%$ à $95,1 \%$ des cas. Alors que pour Mankollo Bassong OY et al. les femmes savaient que le saignement était un signe de danger chez la femme après l'accouchement, $40,21 \%$ pensaient plutôt que c'était la fièvre; $79,72 \%$ l'HTA; 10,84\% les pertes vaginales; 22,03\% l'infection mammaire et $23,43 \%$ l'œdème et la rougeur du mollet ; dans cette même étude, $58,74 \%$ pensaient que la difficulté à téter ou à boire étaient un signe de danger pour le bébé; $30,07 \%$ optaient pour la fièvre ou l'hypothermie; $70,63 \%$ pour la respiration rapide ou difficile; $25,17 \%$ pour l'ictère; 32,87 pour diarrhée/vomissement; et enfin $30,07 \%$ pour la présence du sang dans les selles (16).

La bonne position des mères et celle des nouveau-nés au cours de l'allaitement étaient connues respectivement dans $80,3 \%$ et $78,7 \%$ des cas à l'évaluation initiale contre $91,8 \%$ pour les deux cas à l'évaluation finale. La connaissance sur l'efficacité de la succion du nouveau-né par les mères était $80,3 \%$ contre $82 \%$ des cas respectivement dans l'évaluation initiale et secondaire. Ces bonnes pratiques permettraient d'éviter des complications pour la mère d'une part et un allaitement efficace et sans danger pour le bébé d'autre part.

Nous avons observé un gain des femmes qui étaient très satisfaites pour le service de la CPON de $62,7 \%$ à $80,3 \%$ des cas. Ce gain de satisfaction s'expliquerait par la formation du personnel et leur accompagnement entre les deux évaluations. Alors que la moitié des femmes n'était pas satisfaites du déroulement de la CPON dans le travail d'Assarag $B$ et al., ce résultat était inférieur des deux évaluations de notre étude (17).

\section{Conclusion}

Néanmoins le plan d'action a permis de corriger les insuffisances observées au cours de l'évaluation initiale et d'améliorer la compétence des agents de santé et d'améliorer le niveau de connaissance des femmes.

Conflit d'intérêt : Aucun

\section{Références}

1. Elkhoudri N, Baali A, Amor H. Postnatal Care: Levels and Determinants in Morocco Iranian Journal of Public Health 2017 ; 46(2) : 242-248.

2. Bernard MR, Eymard C. L'éducation pour la santé en périnatalité : enquête auprès des sages-femmes françaises. Santé publique $2014 ; 26$ : 591 à 602.

3. Ronsmans C, Graham WJ. Maternal mortality: who, when, where, and why. Lancet $2006 ; 368$ : 1189-1200.

4. Barlow P, Ceysens $\mathbf{G}$, Emonts $\mathbf{P}$, Gilbert L, Haumont D, Hernandez A et al. Guide du post partum. De Boeck supérieur, Belgique, 2016. ISBN : 978-2-8073-0305-8.

5. Dumon B, Bernard P. Le désir sexuel dans le postpartum. Louvain médical $2010 ; 129$ : S78-S81.

6. WHO. The World Health Report. Make every mother and child count. Geneva, 2005. World Health Organization.

http://www.who.int/whr/2005/whr2005_en.pdf

7. Sénat MV, Sentilhes L, Battut A, Benhamou D, Bydlowski S, Chantry A et al. Post-partum : recommandations pour la pratique clinique - Texte court. Journal de Gynécologie Obstétrique et biologie de la réproduction $2015 ; 44$ : 1157-1166. 
8. Tari C, Riquet S. L'entretien post natal précoce, un nouveau temps pour ajuster le diagnostic éducatif en éducation périnatale. Educ Ther Patient/Ther Patient Educ 2016 ; 8(2) : 20105.

9. Institut National de la Statistique (INSTAT), Cellule de Planification et de Statistique Secteur Santé-Développement Social et Promotion de la Famille (CPS/SS-DS-PF) et ICF. 2019. Enquête Démographique et de Santé au Mali 2018: Rapport de synthèse. Bamako, Mali et Rockville, Maryland, USA : INSTAT, CPS/SS-DS-PF et ICF.

10. Procédure en sante de la reproduction au Mali. Gravido-puerperalité : Soins prénatals, soins pernatals et soins postnatals. Volume 3, juin 2019.

11. Koné C S. Évaluation de la qualité de la consultation postnatale au centre de santé de référence de la commune II de Bamako. FMOS, thes med, 2020.

12. Doret M. Modalités de la consultation postnatale et spécificités en cas de pathologie de la grossesse : recommandations pour la pratique clinique. Journal de gynécologie obstétrique et biologie de la reproduction $2015 ; 44$ : 1118-1126.

13. Maamri A, Badri T, Boujemla H, EL Kissi Y. La sexualité durant le post-partum : étude d'une population de femmes Tunisiennes. La tunisie Medicale $2019 ; 97: 704-710$.

14. Blangis $F$, Lopes $P$, Branger $B$, Garnier $P$, Philippe H J, Ploteau S. La contraception du postpartum : à propos de 600 patientes dont 129 revues à la consultation post-natale. Gynécologie Obstétrique \& Fertilité $2013 ; 41: 499-504$.

15. La revue du praticien. Complication du post partum. Revue du praticien Médecine Générale $2018 ; 32$ (1002) : 419-25.

16. Mankollo Bassong $0 \mathrm{Y}$, Djeumo Djatcha $\mathrm{J} \mathrm{L}$, Tchinda Fossi C, Fossi A. Connaissances et Représentations Sociales du Suivi Postnatal dans le District de Santéde Bafang (Cameroun). Health Sci. Dis $2020 ; 21$ (5) : 41-46.

17. Assarag B, Nassiri K, Kharbach A. Les déterminants de l'utilisation de la consultation postnatale à la préfecture de Skhirat-Témara, Maroc. Révue d'Epidémiologie et de santé publique $2014 ; 62$ : S175-S176.

Liste des tableaux et figures

\section{Qualification du personnel soignant}

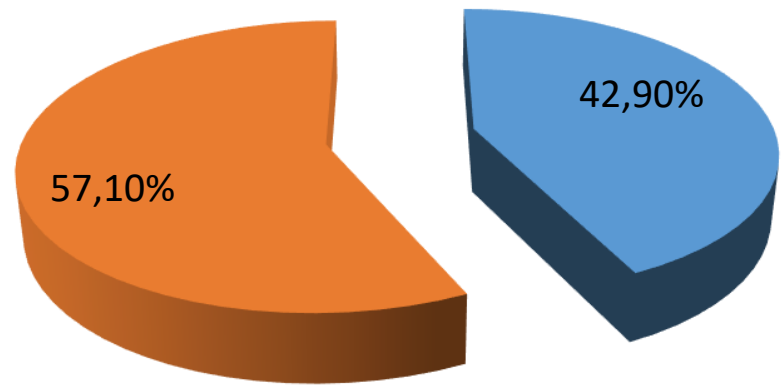

Presonnel qualifié (Sage femme + infirmière obstétricienne)

Presonnel non qualifié ( matrone)

Figure 1 : Qualification du personnel soignant 


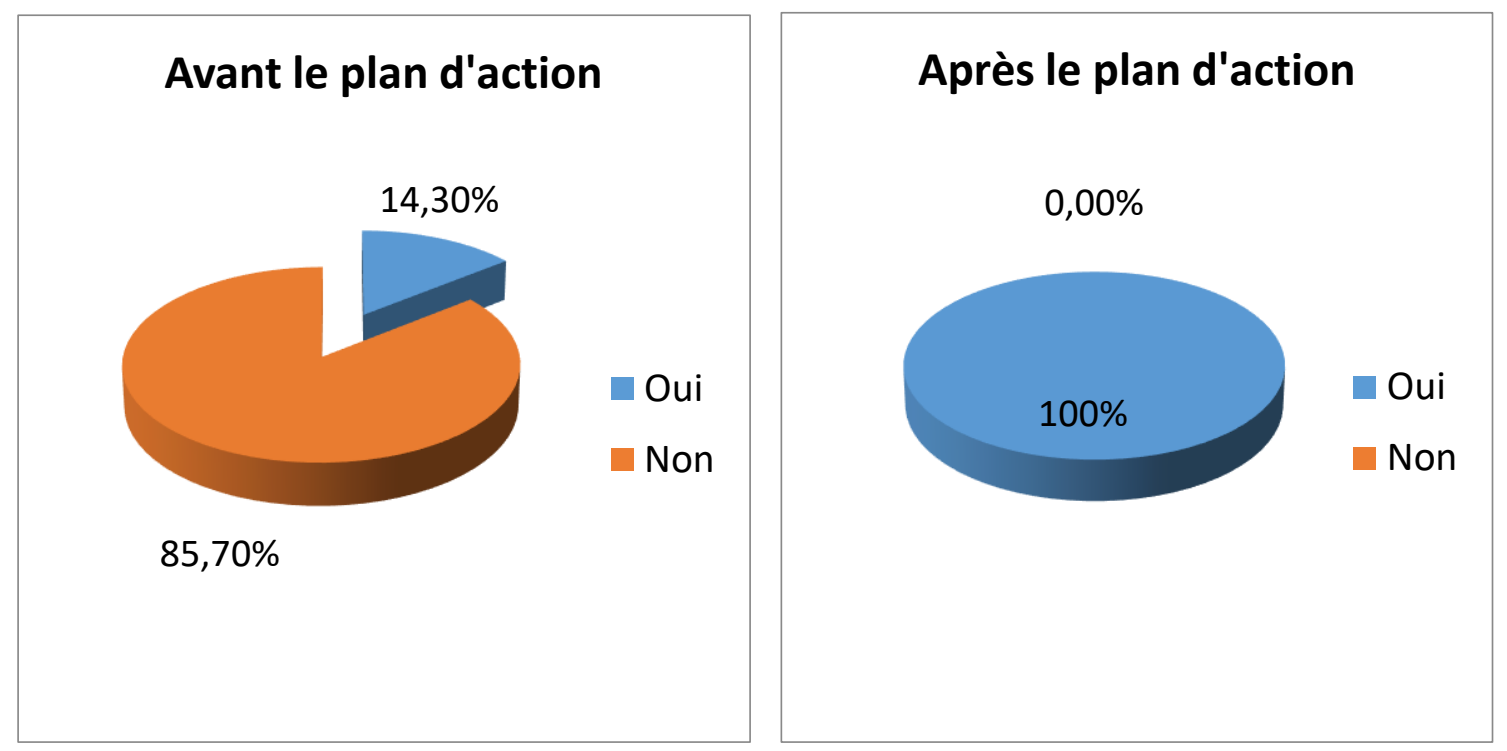

Figure 2 : Réalisation de l'examen du nouveau-né au cours de la CPON

Tableau I : Caractéristiques sociodémographiques des femmes en post partum

\begin{tabular}{lcccc}
\hline & \multicolumn{2}{c}{ Avant } & & Après \\
\cline { 2 - 5 } & Effectif & Pourcentage & Effectif & Pourcentage \\
\hline Tranche d'âge & & & 22 & 18 \\
1619 ans & 28 & 23,0 & 64 & 52,5 \\
2029 ans & 58 & 47,5 & 34 & 27,9 \\
3039 ans & 28 & 23,0 & 2 & 1,6 \\
40 49 ans & 8 & 6,6 & 112 & 91,8 \\
Provenance & & & 10 & 8,2 \\
Rayon 5 km & 116 & 95,1 & & 57,4 \\
Supérieur 15 km & 6 & 4,9 & 40 & 39,3 \\
Niveau de scolarisation & & & 48 & 3,3 \\
Non scolarisé & 64 & 52,5 & 4 & 18 \\
Primaire & 50 & 41,0 & & 36,1 \\
Secondaire & 8 & 6,6 & 22 & 37,7 \\
Parité & & & 44 & 6,6 \\
Primipare & 28 & 22,9 & 46 & 1,6 \\
Paucipare & 44 & 36,1 & 2 & \\
Multipare & 34 & 27,9 & 11,5 & \\
Grande multipare & 14 & 1,6 & & \\
Très grande multipare & 2 & & & \\
\hline
\end{tabular}


Tableau II : Connaissance des mères sur les signes de danger chez les femmes en post partum et chez les nouveaunés

\begin{tabular}{lcccc}
\hline & \multicolumn{2}{c}{ Avant } & \multicolumn{2}{c}{ Après } \\
\cline { 2 - 5 } & Effectif & Pourcentage & Effectif & Pourcentage \\
\hline Connaissance de signes de danger & chez les femmes en post partum & & \\
Oui & 82 & 67,2 & 112 & 91,8 \\
Non & 40 & 32,8 & 10 & 8,2 \\
Connaissance de signes de danger chez les nouveau-nés & 65,6 & 112 & 91,8 \\
Oui & 80 & 34,4 & 10 & 8,2 \\
Non & 42 & 100 & 122 & 100 \\
Total & 122 & & & \\
\hline
\end{tabular}

Tableau III : Satisfaction des mères sur la CPON

\begin{tabular}{lcccc} 
& \multicolumn{2}{c}{ Avant } & \multicolumn{2}{c}{ Après } \\
\cline { 2 - 5 } & Effectif & Pourcentage & Effectif & Pourcentage \\
\hline Pas satisfaite & 14 & 11,5 & 0 & 0 \\
Peu satisfaite & 32 & 26,2 & 24 & 19,7 \\
Très satisfaite & 76 & 62,3 & 98 & 80,3 \\
Total & 122 & 100 & 122 & 100 \\
\hline
\end{tabular}

